\title{
ON THE RICCI CURVATURE OF A HYPERSURFACE IN A SPHERE
}

\author{
MEHMET ERDOĞAN
}

We know a lot of results concerning Ricci curvature of hypersurfaces in a space form. In 1983 P. F. Leung ([3]) gave the interesting estimate of Ricci curvature of a complete hypersurface in an $(n+1)$-dimensional Euclidean space $E^{n+1}$ as follows;

Proposition ([3]). Let $M$ be a complete hypersurface in $E^{n+1}$ such that all sectional curvatures of $M$ are bounded away from $-\infty$. If $M$ is contained in a ball of radius $r$, then

$$
\lim \sup _{\substack{p \in M \\ X \in T_{p} M \\\|X\|=1}} \operatorname{Ric}(X, X) \geqq \frac{n-1}{r^{2}} .
$$

In this paper, we will extend Leung's result from the Euclidean case to the spherical case. That is, we will prove the following

THEOREM. Let $M$ be a complete, noncompact, n-hypersurface in the unit $(n+1)$-sphere $S^{n+1}$ in $E^{n+2}$ such that all sectional curvatures of $M$ are bounded away from $-\infty$. If $M$ is contained in a geodesic ball of radius $r$ and if we assume that $r$ is less than $\pi / 2$, then we have

$$
\lim \sup _{\substack{p \in M \\ X \in T_{T} M \\\|X\|=1}} \operatorname{Ric}(X, X) \geqq n-1+\frac{(n-1) \cos ^{2} r}{4 \sin ^{2}(r / 2)} .
$$

In order to prove theorem above, we use the following useful results.

Lemma 1 ([4]). Let $M$ be a complete, noncompact and connected Riemannian manifold whose sectional curvatures are bounded away from $-\infty$. Let $f$ be a smooth and bounded function on $M$. Then, for any $\varepsilon>0$, there is a point

Received February 5, 1996. 
$p \varepsilon M$ such that $\|\operatorname{grad} f(p)\|<\varepsilon$ and $H_{f}(X, X)<\varepsilon$ for any unit vector $X \varepsilon T_{P} M$, where $H_{f}$ denotes the Hessian of $f$.

Let $\sigma: E^{n} \times E^{n} \rightarrow E^{k}$ be a symmetric bilinear form and consider the real valued function $\Phi(X)=\|\sigma(X, X)\|^{2}: S^{n-1} \rightarrow R$, where $S^{n-1}$ is a unit sphere in $E^{n}$. Since $\Phi$ is differentiable and $S^{n-1}$ is compact, $\Phi$ attains its minimum at some point $X_{0} \in S^{n-1}$. Considering $\sigma\left(X_{0}, *\right): E^{n} \rightarrow E^{k}$ as a linear transformation, we have

Lemma 2 ([5]). Suppose that $\sigma\left(X_{0}, X_{0}\right) \neq 0$. Then we have

(1) $X_{0} \perp \operatorname{Ker} \sigma\left(X_{0}, *\right)$,

(2) $\left\langle\sigma\left(X_{0}, X_{0}\right), \sigma(Y, Y)\right\rangle \geq\left\|\sigma\left(X_{0}, X_{0}\right)\right\|^{2}$, for any unit vector $Y \varepsilon \operatorname{Ker} \sigma\left(X_{0}, *\right)$.

\section{The proof of Theorem.}

Let $S^{n+1}$ be the unit $(\mathrm{n}+1)$-sphere in $E^{n+2}$ defined by

$$
S^{n+1}=\left\{x \in E^{n+2}:\|x\|=1\right\} .
$$

At each point $\boldsymbol{p} \in S^{n+1}$ we take the unit normal vector $\xi=-p$ to $S^{n+1}$ in $E^{n+2}$. Denote $\hat{\nabla}$ and $\tilde{\nabla}$ the covariant derivative of $E^{n+2}$ and $S^{n+1}$ respectively, then we have the formula of Gauss

$$
\hat{\nabla}_{x} Y=\tilde{\nabla}_{X} Y-\langle X, Y\rangle \boldsymbol{p},
$$

where $X$ and $Y$ are vector fields tangent to $S^{n+1}$ at $\boldsymbol{p}$.

Now we consider a hypersurface $M$ in $S^{n+1}$, that is, an $n$-dimensional Riemannian manifold $M$ embedded in $S^{n+1}$ with induced Riemannian structure. We denote $\nabla$ the covariant derivative of $M$. Then, for tangent vector fields $X, Y$ on $M$, the formula of Gauss is

$$
\hat{\nabla}_{X} Y=\tilde{\nabla}_{X} Y+\sigma(X, Y),
$$

where $\sigma$ is the second fundamental form of $M$ which is a symmetric bilinear form of $T M \times T M$ to $N M$, where $T M$ and $N M$ are the tangent bundle and the normal bundle of $M \subset S^{n+1}$ respectively. For a plane section $P$ spanned by the pair of orthonormal vectors $X, Y \in T M$, the sectional curvature $K(X, Y)$ of $M$ is written as

$$
K(X, Y)=1+\langle\sigma(X, X), \sigma(Y, Y)\rangle-\|\sigma(X, Y)\|^{2} .
$$

Let $\left\{X, Y_{1}, Y_{2}, \ldots, Y_{n-1}\right\}$ be an orthonormal basis of $T M$, then the Ricci curvature in $X$-direction is given by 


$$
\operatorname{Ric}(X, X)=\sum_{i=1}^{n-1} K\left(X, Y_{i}\right) .
$$

It follows from (1.3) and (1.4) that we have

$$
\operatorname{Ric}(X, X)=n-1+\sum_{i=1}^{n-1}\left\{\left\langle\sigma(X, X), \sigma\left(Y_{i}, Y_{i}\right)\right\rangle-\left\|\sigma\left(X, Y_{i}\right)\right\|^{2}\right\} .
$$

All hypersurfaces in $S^{n+1}$ are bounded, that is, $M$ is contained in a closed geodesic ball of finite radius $r$. Without loss of generality we take such a geodesic ball as the closed ball $B(\boldsymbol{a}, r)$ with the center $\boldsymbol{a}=(0,0, \ldots, 1)$. By simple trigonometry, we see that the distance in $E^{n+2}$ from $\boldsymbol{a}$ to a point of the geodesic sphere $S(\boldsymbol{a}, r)=\partial B(\boldsymbol{a}, r)$ is $2 \sin (r / 2)=\lambda$.

Now, we consider the real-valued function $f(\boldsymbol{x})=\langle\boldsymbol{x}-\boldsymbol{a}, \boldsymbol{x}-\boldsymbol{a}\rangle: M \rightarrow R$. We have

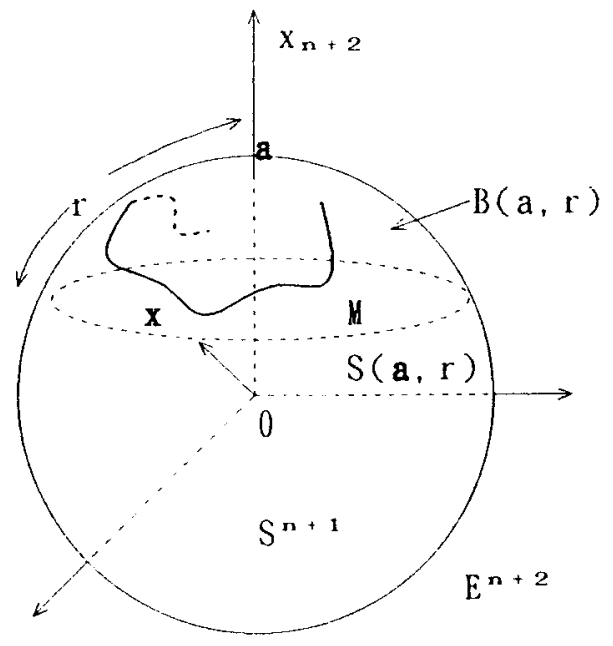

$$
f(\boldsymbol{x})=\|\boldsymbol{x}-\boldsymbol{a}\|^{2} \leq \lambda^{2} .
$$

Then, the Hessian $H_{f}(X, X)$ of $f$ at a point $x \in M$ for a unit tangent vector $X \in T_{x} M$ is

$$
\begin{aligned}
H_{f}(X, X) & =X(X\langle\boldsymbol{x}-\boldsymbol{a}, \boldsymbol{x}-\boldsymbol{a}\rangle)-\left(\nabla_{X} X\right)\langle\boldsymbol{x}-\boldsymbol{a}, \boldsymbol{x}-\boldsymbol{a}\rangle \\
& =2 X\langle X, \boldsymbol{x}-\boldsymbol{a}\rangle-2\left\langle\nabla_{X} X, \boldsymbol{x}-\boldsymbol{a}\right\rangle \\
& =2\left\langle\hat{\nabla}_{X} X, \boldsymbol{x}-\boldsymbol{a}\right\rangle+2\langle X, X\rangle-2\left\langle\nabla_{X} X, \boldsymbol{x}-\boldsymbol{a}\right\rangle,
\end{aligned}
$$


which, together with (1.1) and (1.2), implies

$$
H_{f}(X, X)=2\langle\sigma(X, X), \boldsymbol{x}-\boldsymbol{a}\rangle+2-2\langle\boldsymbol{x}, \boldsymbol{x}-\boldsymbol{a}\rangle .
$$

From (1.7) we have

$$
\begin{aligned}
\|\sigma(X, X)\| & \geq \frac{\|\mathbf{x}-\mathbf{a}\|}{\lambda}\|\sigma(x, x)\| \geq-\frac{1}{2 \lambda}\{2\langle\sigma(X, X), \boldsymbol{x}-\boldsymbol{a}\rangle\} \\
& =\frac{1}{2 \lambda}\left(2-H_{f}(X, X)\right)-\frac{1}{\lambda}\langle\boldsymbol{x}, \boldsymbol{x}-\boldsymbol{a}\rangle .
\end{aligned}
$$

By Lemma 1, for any integer $m$, we have a point $x \in M$ such that $H_{f}(X, X)<2 / m$ for all unit tangent vector $X \in T_{x} M$. We have $\langle\boldsymbol{x}, \boldsymbol{x}-\boldsymbol{a}\rangle=\langle\boldsymbol{x}, \boldsymbol{x}\rangle-\langle\boldsymbol{x}, \boldsymbol{a}\rangle=1-\cos \theta$, where $\theta$ is the distance form $\boldsymbol{x}$ to a on $S^{n+1}$. Since $\cos \theta \geq \cos r$, from (1.8) we get

$$
\|\sigma(X, X)\|>\frac{1}{\lambda}\left(1-\frac{1}{m}\right)+\frac{-1+\cos r}{\lambda},
$$

which implies

$$
\|\sigma(X, X)\|>\frac{1}{\lambda}\left(1-\frac{1}{m}\right)-\frac{\lambda}{2},
$$

because $-1+\cos r=-\lambda^{2} / 2$. Since $\lambda=2 \sin (r / 2) \leq 2 \sin (\pi / 4)=\sqrt{2}$, for large $m$ we may assume that

$$
\frac{1}{\lambda}\left(1-\frac{1}{m}\right)-\frac{\lambda}{2}>0,
$$

which, together with (1.9), implies that $\sigma(X, X) \neq 0$ for all unit tangent vector $X \in T_{x} M$.

Let $X_{0}$ be the unit tangent vector to $M$ at $x \in M$ such that $\left\|\sigma\left(X_{0}, X_{0}\right)\right\|^{2}$ is the minimum of all $\|\sigma(X, X)\|^{2}, \quad X \in T_{x} M$. Since $\sigma\left(X_{0}, X_{0}\right) \neq 0, X_{0} \notin$ $\operatorname{Ker} \sigma\left(X_{0}, *\right)$, we have

$$
\operatorname{dimKer} \sigma\left(X_{0}, *\right)+\operatorname{dim} \operatorname{Im} \sigma\left(X_{0}, *\right)=\operatorname{dim} T_{x} M,
$$

and so we get

$$
\operatorname{dimKer} \sigma\left(X_{0}, *\right)=n-1,
$$

which implies that we have an orthonormal basis $\left\{X_{0}, X_{1}, \ldots, X_{n-1}\right\}$ of $T_{x} M$ satisfying $\sigma\left(X_{0}, X_{i}\right)=0,1 \leq i \leq n-1$.

It follows from (1.5) and (2) of Lemma 2 that we have 


$$
\begin{aligned}
\operatorname{Ric}\left(X_{0}, X_{0}\right) & =n-1+\sum_{i=1}^{n-1}\left\langle\sigma\left(X_{0}, X_{0}\right), \sigma\left(X_{i}, X_{i}\right)\right. \\
& \geq n-1+\sum_{i=1}^{n-1}\left\|\sigma\left(X_{0}, X_{0}\right)\right\|^{2}
\end{aligned}
$$

which, together with $(1,9)$, implies

$$
\operatorname{Ric}\left(X_{0}, X_{0}\right)>n-1+(n-1)\left(\frac{1}{\lambda}\left(1-\frac{1}{m}\right)-\frac{\lambda}{2}\right)^{2} .
$$

Letting $m \rightarrow \infty$, we have

$$
\lim \sup _{\substack{p \in M \\ X \in T_{p} M \\\|X\|=1}} \operatorname{Ric}(X, X) \geq(n-1)+(n-1)\left(\frac{1}{\lambda}-\frac{\lambda}{2}\right)^{2} .
$$

Since $\lambda=2 \sin (r / 2)$, from (1.11) we have the inequality of theorem.

\section{REFERENCES}

1. S. S. Chern and R. K. Lashof, On the total curvature of immersed manifolds I, Amer. J. Math. 79 (1957), 306-318; II, Michigan Math. J. 5(1958), 5-12.

2. S. Kobayashi and K. Nomizu, Foundations of Differential Geometry II, Interscience Tracts., New York, London, Sydney, 1969.

3. P. F. Leung, Complete hypersurfaces of non-positive Ricci curvature, Bull. Austra. Math. Soc. 27 (1983), 215-219.

4. H. Omori, Isometric immersions of Riemannian manifolds, J. Math. Soc. Japan (1967), 205214.

5. M. Spivak, A comprehensive introduction to differential geometry, Volume 5, Page 193, Publish or Perish, Boston, Massachusetts, (1975).

DEPARTMENT OF MATHEMATICS

FACULTY OF SCIENCE

ISTANBUL UNIVERSITY

34459 VEZNECILER, ISTANBUL

TURKEY 


\title{
ON SINGULARITIES OF FOLDING MAPS AND AUGMENTATIONS
}

\author{
KEVIN HOUSTON
}

\section{Introduction.}

In this paper we shall investigate the properties of two different types of map germs of the form $f:\left(\mathrm{C}^{n}, S\right) \rightarrow\left(\mathrm{C}^{p}, 0\right)$ where $S$ is a finite set of points of $\mathrm{C}^{n}$. The first is the fold type map, which is a map of the form,

$$
\left(x_{1}, \ldots, x_{n-1}, y\right) \mapsto\left(x_{1}, \ldots, x_{n-1}, y^{2}, y h_{1}\left(x, y^{2}\right), \ldots y h_{p-n}\left(x, y^{2}\right)\right),
$$

for some holomorphic functions $h_{i}, i=1, \ldots, p-n$. These first arose in the classification of map germs $f:\left(\mathrm{C}^{2}, 0\right) \rightarrow\left(\mathrm{C}^{3}, 0\right)$ by Mond, [15], and were later studied by Wilkinson, [19]. These maps are connected with the classification of isolated complete intersections with respect to diffeomorphisms that preserve a certain linear subspace of $\mathrm{C}^{n}$.

In [12] there is defined a topological invariant of the map germ, called a disentanglement which is analogous to the Milnor fibre of an isolated complete intersection singularity. In the case of folding maps the disentanglement is homotopically equivalent to a wedge of spheres of dimension $2 n-p+1$.Denote this number by $\mu_{I}(f)$ and call it the image Milnor number of $f$.

The main result for folding maps, Theorem 2.7 , is that for $f$ finitely $\mathscr{A}$ determined, (see,for example, [1] for definitions), with $p<2 n-1$ we have,

$$
\mathscr{A}_{e} \text {-codimension }(f) \leq \mu_{I}(f) \text {. }
$$

If $p=n+1$ and $f$ is quasihomogeneous then this is actually an equality. David Mond has informed me that he has proved this particular case via another method. Examples show that for the case $p=2 n$ a similar statement to the above is false. The case $p=2 n-1$ is not investigated.

The second type of map is the augmentation of a map germ. For a map $f$

Received February 12, 1996. 
unfolded with $F(x, t)=\left(f_{t}(x), t\right)$, take a complex analytic function germ $g:\left(\mathrm{C}^{q}, 0\right) \rightarrow(\mathrm{C}, 0)$ and define a new map through $A_{F, g}(f)=\left(f_{g(z)}(x), z\right)$.

This is a generalisation of the definition in [3] where $f$ has $\mathscr{A}_{e}$-codimension one, $F$ is the $\mathscr{A}_{e}$-versal unfolding of $f$ and $g(z)=z^{2}$.

The main result for augmentations, Theorem 3.2, is that if $F$ is stable (i.e. its $\mathscr{A}_{e}$-codimension is zero), then

$$
\tau(g) . \mathscr{A}_{e} \text {-codimension }(f) \leq \mathscr{A}_{e} \text {-codimension }\left(A_{F, g}(f)\right),
$$

where $\tau(g)$ denotes the Tjurina number of $g$. If $F$ is in the nice dimensions or $g$ is quasihomogeneous, then there is equality.

As we shall see, folding maps provide a source of examples of augmentations and augmentations provide new examples of series of singularities.

For general references on singularity theory, e.g. definition of $\mathscr{A}$ and $\mathscr{K}$ equivalence, etc., see [1], [2] and [17]. For a map $H: \mathrm{C}^{n} \rightarrow \mathrm{C}^{p}$, let $X=H^{-1}(0)$ and denote the Milnor number and Tjurina numbers with $\mu(H)$ or $\mu(X)$ and $\tau(H)$ or $\tau(X)$ respectively.

Since in the statement of theorems we shall be interested in $\mathscr{A}$-equivalence of a map germ $f$ we shall denote $\mathscr{A}_{e}$-codimension with $\operatorname{cod}(f)$. Other codimensions will be written explicitly.

My thanks to Bill Bruce and Neil Kirk for helpful discussions and comments. I am particularly grateful to Mihai Tibar for pointing out an error in Theorem 2.8 in an earlier version of the paper and to the referee for suggesting a number of improvements. The author was supported by a grant from EPSRC (Grant number GR/K/29227) during the writing of this paper. The computer program Singular was used in calculating a number of the examples in this paper.

\section{Folding Maps.}

In this section we shall study the algebraic codimension and the local topology of folding maps.

We use the following notation. For the ring of germs from $\mathrm{C}^{n}$ to $\mathrm{C}$, usually denoted $\mathcal{O}_{\mathrm{C}^{n}}$ we use $\mathcal{O}_{n} ; \mathcal{O}_{n}^{p}$ stands for $\oplus_{i=1}^{p} \mathcal{O}_{n}$. Coordinates on $\mathrm{C}^{n}$ will be given by $\left(x_{1}, \ldots, x_{n-1}, y\right)$. For a map $h: \mathrm{C}^{n} \rightarrow \mathrm{C}^{p}$ denote by $I_{h}$ the ideal generated in $\mathcal{O}_{n}$ by the coordinate functions: $I_{h}=\left\langle h_{1}, \ldots, h_{p}\right\rangle$. The phrase isolated complete intersection singularity is abbreviated to ICIS.

Let $h: \mathrm{C}^{n} \rightarrow \mathrm{C}^{p-n}$ be given by $\left(h_{1}(x, y), \ldots, h_{p-n}(x, y)\right)$.

Let $H: \mathrm{C}^{n} \rightarrow \mathrm{C}^{p-n}$ be given by $\left(h_{1}\left(x, y^{2}\right), \ldots, h_{p-n}\left(x, y^{2}\right)\right)$.

Let $H^{\prime}: \mathrm{C}^{n} \rightarrow \mathrm{C}^{p-n+1}$ be given by $\left(h_{1}\left(x, y^{2}\right), \ldots, h_{p-n}\left(x, y^{2}\right), y\right)$.

Definition 2.1. Suppose $f:\left(\mathrm{C}^{n}, 0\right) \rightarrow\left(\mathrm{C}^{p}, 0\right)$, with $1<n<p$, is a map germ. Then $f$ is called a folding map if it is $\mathscr{A}$-equivalent to a map of the form, 


$$
\left(x_{1}, \ldots, x_{n-1}, y\right) \mapsto\left(x_{1}, \ldots, x_{n-1}, y^{2}, y h_{1}\left(x, y^{2}\right), \ldots, y h_{p-n}\left(x, y^{2}\right)\right),
$$

for some functions $h_{i}(x, y)$, for $i=1, \ldots, p-n$.

ExAmPLes 2.2. (i) The map $f(x, y)=\left(x, y^{2}\right)$ is the archetypal folding map.

(ii) It is well known, see [18], that the stable map germs $f:\left(\mathrm{C}^{n}, 0\right) \rightarrow\left(\mathrm{C}^{2 n-1}, 0\right)$ which are not immersions are of the form

$$
f\left(x_{1}, \ldots, x_{n-1}, y\right)=\left(x_{1}, \ldots, x_{n-1}, y^{2}, y x_{1}, \ldots, y x_{n-1}\right) .
$$

Proposition 2.3. Suppose $f$ is a folding map. Then $f$ is finitely $\mathscr{A}$-determined if and only if $H$ is finitely $\mathscr{K}$-determined. If $f$ is finitely $\mathscr{A}$-determined then $H^{\prime}: \mathrm{C}^{n-1} \rightarrow \mathrm{C}^{p-n}$ is finitely $\mathscr{K}$-determined.

Proof. Both results come from the characterisation of corank 1 map germs by Marar and Mond in [13]. In their Theorem 2.14 they prove that a corank 1 map germ $f$ is finitely $\mathscr{A}$-determined if and only if the multiple points spaces are ICIS, or empty. Furthermore, in Corollary 2.15 of the same paper they prove that if $f$ is finitely $\mathscr{A}$-determined then the restriction to the fixed point sets of the elements of $S_{k}$ in $D^{k}(f)$ also define ICIS. (The $k^{\text {th }}$ multiple point space of $f$ is the closure in $X^{k}$ of the set of distinct $k$-tuples that have the same image under $f$ ).

For $D^{k}(f)$ to be non-empty there must be distinct $y_{i}$ such that $y_{1}^{2}=y_{2}^{2}=\ldots=y_{k}^{2}$, but this is never satisfied if $k>2$.Thus the results rest on proving that the double point space, $D^{2}(f)$ is defined by $H$, and that the fixed point set of the non-trivial element of $S_{2}$ is defined by $H^{\prime}$. These are easily seen to be true.

We now state the definition of a disentanglement of a map germ, see [12] and [9].

Definition 2.4. Let $f:\left(\mathrm{C}^{n}, S\right) \rightarrow\left(\mathrm{C}^{p}, 0\right)$ be a map germ with $n<p$ and $S$ a finite set. Suppose $F:\left(\mathrm{C}^{n} \times \mathrm{C}^{r}, S \times 0\right) \rightarrow\left(\mathrm{C}^{p} \times \mathrm{C}^{r}, 0 \times 0\right)$ is an r-parameter unfolding of $f$. Denote $F(x, t)$ by $f_{t}(x)$.

Let $B_{\epsilon}$ be a small ball centred at zero in $\mathrm{C}^{p} \times \mathrm{C}^{r}$. Let $U=F^{-1}\left(B_{\epsilon}\right)$ and $U_{t}=U \cap F^{-1}\left(C^{p} \times\{t\}\right)$, where $\epsilon$ is chosen so small that $S_{\epsilon^{\prime}}$ is stratified transverse to $F(U)$ for all $\epsilon^{\prime} \leq \epsilon$.

The image of $f_{t} \mid U_{t}$ is called a disentanglement of $f$. If $f_{t}$ is (topologically) stable then the image of $f_{t} \mid U_{t}$ is called a (toplogically) stable disentanglement.

REMARK 2.5 . If $f$ is finitely $\mathscr{A}$-determined then topologically stable disentanglements always exist and are essentially unique. If $(n, p)$ is in the nice dimensions then stable disentanglements always exist. Since Milnor fibres of ICIS exist, Proposition 2.2 tells us that stable disentanglements always exist for folding maps.

A disentanglement of $f$ is the analogue of a Milnor fibre for a map-germ 
$g:\left(\mathrm{C}^{n}, 0\right) \rightarrow\left(\mathrm{C}^{p}, 0\right)$ with $n \geq p$. Milnor fibres are non-singular; however, the disentanglement of $f$ can be very singular, since typically the image will intersect itself along a subspace of dimension $2 n-p$. Furthermore, the number of equations defining the image of $F$ in $\mathrm{C}^{p} \times \mathrm{C}^{d}$ can be very large. For $p=n+1$ the image is a hypersurface but in general it is not even a complete intersection.

Theorem 2.6. Suppose $f:\left(\mathrm{C}^{n}, 0\right) \rightarrow\left(\mathrm{C}^{p}, 0\right)$, with $1<n<p \leq 2 n$, is a $f$ nitely $\mathscr{A}$-determined folding map then the disentanglement of $f$ is homotopically equivalent to a wedge of spheres of dimension $2 n-p+1$.

Proof. By [9] Theorem 3.24 the disentanglement of $f$ is a wedge of spheres (of possibly different dimensions). Since $D^{k}(f)=\emptyset$ for $k>2$, by Corollary 3.8 of [9] we have

$$
\tilde{H}_{i}\left(f_{t}\left(U_{t}\right) ; \mathrm{Q}\right) \cong \text { Alt } \mathrm{H}_{i+1}\left(D^{2}(f) ; \mathrm{Q}\right)
$$

and the only non-zero alternating homology group is $\mathrm{Alt}_{\mathrm{dim} D^{2}(f)}\left(D^{2}(f) ; \mathrm{Q}\right)$. As $\operatorname{dim} D^{2}(f)=2 n-p$ the result follows.

Following Mond in [16] we shall call the number of spheres occurring in the wedge the image Milnor number of $f$ and we denote it by $\mu_{I}(f)$ or $\mu_{I}$.

For the case of finitely $\mathscr{A}$-determined maps germs in the nice dimensions with $p=n+1$, Mond has proved that the disentanglement is a wedge of $n$ spheres.

Conjecture 2.7. Mond has conjectured that the number of spheres in the disentanglement is greater than or equal to the $\mathscr{A}_{e}$-codimension of the germ, with equality if the germ is quasihomogeneous.

This conjecture has been proved if $n=2$ and $p=3$ in [10] and [16].

In Theorem 2.11 we show that a similar result on the the number of vanishing cycles versus codimension holds for folding maps with $p<2 n-1$.

The next theorem allows us to calculate the $\mathscr{A}_{e}$-codimension of a folding map with a formula similar to the one for the $\mathscr{K}_{e}$-codimension of an ICIS. This is a generalisation of Example 1.1 in [4].

Theorem 2.8. Suppose $f$ is a folding map. Then

$$
\operatorname{cod}(f)=\operatorname{dim}_{\mathrm{C}} \frac{\mathcal{O}_{n}^{p-n}}{\left\langle y \frac{\partial h}{\partial y}, \frac{\partial h}{\partial x_{1}} \cdots \frac{\partial h}{\partial x_{n-1}}\right\rangle \mathcal{O}_{n}+I_{h} \mathcal{O}_{n}^{p-n}}
$$

Proof. The proof is similar to that of Proposition 4.1:16 of [15]. One can also see the result as a consequence of Theorem 5.2.4 of [19] that two folding 
maps $f$ and $f^{\prime}$ are $\mathscr{A}$-equivalent if and only if $D^{2}(f)$ and $D^{2}\left(f^{\prime}\right)$ are $\mathscr{K}_{\delta^{-}}$ equivalent.

The next lemma relates a formula similar to the one in the above theorem to the Tjurina numbers of the singularities defined by $H$ and $H^{\prime}$.

Lemma 2.9. Recall that $H(x, y)=h\left(x, y^{2}\right)$ and $X=H^{-1}(0)$, then

$$
\tau(X)+\tau\left(\left.X\right|_{y=0}\right) \geq \operatorname{dim}_{\mathrm{C}} \frac{\mathcal{O}_{n}^{p-n}}{\left\langle y \frac{\partial H}{\partial y}, \frac{\partial H}{\partial x_{1}}, \ldots, \frac{\partial H}{\partial x_{n-1}}\right\rangle \mathcal{O}_{n}+I_{H} \mathcal{O}_{n}^{p-n}} .
$$

Proof. Consider the following sequence,

$$
\begin{aligned}
& \frac{\mathcal{O}_{n}^{p-n}}{\left\langle\frac{\partial H}{\partial y}, \frac{\partial H}{\partial x_{1}}, \ldots, \frac{\partial H}{\partial x_{n-1}}\right\rangle \mathcal{O}_{n}+I_{H} \mathcal{O}_{n}^{p-n}} \stackrel{. y}{\rightarrow} \\
& \frac{\mathcal{O}_{n}^{p-n}}{\left\langle y \frac{\partial H}{\partial y}, \frac{\partial H}{\partial x_{1}}, \ldots, \frac{\partial H}{\partial x_{n-1}}\right\rangle \mathcal{O}_{n}+I_{H} \mathcal{O}_{n}^{p-n}} \stackrel{\text { pr }}{\longrightarrow} \\
& \frac{\mathcal{O}_{n}^{p-n}}{\left\langle\frac{\partial H}{\partial y}, \frac{\partial H}{\partial x_{1}}, \ldots, \frac{\partial H}{\partial x_{n-1}}\right\rangle \mathcal{O}_{n}+\left(I_{H}+\langle y\rangle\right) \mathcal{O}_{n}^{p-n}} \rightarrow 0 .
\end{aligned}
$$

We show that the sequence is exact and relate the terms in it to the terms in the statement of the lemma. The second map is surjective since

$$
\left\langle y \frac{\partial H}{\partial y}, \frac{\partial H}{\partial x_{1}}, \ldots, \frac{\partial H}{\partial x_{n-1}}\right\rangle \mathcal{O}_{n}+I_{H} \mathcal{O}_{n}^{p-n}
$$

is a subset of

$$
\left\langle\frac{\partial H}{\partial y}, \frac{\partial H}{\partial x_{1}}, \ldots, \frac{\partial H}{\partial x_{n-1}}\right\rangle \mathcal{O}_{n}+\left(I_{H}+\langle y\rangle\right) \mathcal{O}_{n}^{p-n} .
$$

But since $H(x, y)=h\left(x, y^{2}\right)$ we get $\frac{\partial H}{\partial y} \in\langle y\rangle \mathcal{O}_{n}^{p-n}$, thus the sequence is exact at the other position.

The first term of the sequence has dimension equal to $\tau(X)$; the third term has dimension equal to $\tau\left(\left.X\right|_{y=0}\right)$. Recall that $H^{\prime}$ is defined by $(H(x, y), y)$, and so $\tau\left(\left.X\right|_{y=0}\right)=\tau\left(H^{\prime-1}(0)\right)$. It is obvious that $\frac{\partial H^{\prime}}{\partial x}=\left[\frac{\partial H}{\partial x} 0\right]^{t}$ and that $\frac{\partial H^{\prime}}{\partial y}=\left[\begin{array}{ll}\frac{\partial H}{\partial y} & 1\end{array}\right]^{t}$. Here the superscript $t$ denotes transpose. 
Since $\frac{\partial H}{\partial y} \in\langle y\rangle \mathcal{O}_{n}^{p-n}$ we have

$$
[0 \ldots 01]^{t} \in\left\langle\frac{\partial H^{\prime}}{\partial x_{1}}, \ldots, \frac{\partial H^{\prime}}{\partial x_{n-1}}, \frac{\partial H^{\prime}}{\partial y}\right\rangle \mathcal{O}_{n}+I_{H^{\prime}} \mathcal{O}_{n}^{p-n+1}
$$

and since $I_{H^{\prime}}=I_{H}+\langle y\rangle$ we deduce that the third term is equal to $\tau\left(\left.X\right|_{y=0}\right)$.

The inequality to prove is then a consequence of the exactness of the sequence above.

REMARKS 2.10. (i) If $p=n+1, H$ is a quasihomogeneous isolated singularity and the restriction of $H$ to $y=0$ has an isolated singularity then the inequality becomes an equality. This is because obviously $\tau(X)=\mu(X)$, $\tau\left(\left.X\right|_{y=0}\right)=\mu\left(\left.X\right|_{y=0}\right)$ and the third term is the boundary Milnor number for $H$. The sum of the two Milnor numbers is well known to equal the boundary number. See [2] p.12.

(ii) The inequality need not be an equality. Take the non-quasihomogeneous example $H(x, y)=y^{8}+x^{3}+y^{6} x$. Here $\tau(X)=13$ and $\tau\left(\left.X\right|_{y=0}\right)=2$ but the remaining term is equal to 14 . I conjecture that if $H$ is quasihomogeneous then the inequality is in fact an equality.

We now reach the main result of this section; a result on the number of vanishing cycles versus the $\mathscr{A}_{e}$-codimension.

Theorem 2.11. Suppose $f:\left(\mathrm{C}^{n}, 0\right) \rightarrow\left(\mathrm{C}^{p}, 0\right)$ is a finitely $\mathscr{A}$-determined folding map such that $p<2 n-1$. Then $\mu_{I}(f) \geq \operatorname{cod}(f)$. If $p=n+1$ and $f$ is quasihomogeneous then $\mu_{I}(f)=\operatorname{cod}(f)$.

Proof. As stated in the proof of Theorem 2.4, the number of vanishing cycles, $\mu_{I}(f)$, is equal to the rank of Alt $\mathrm{H}_{2 n-p}\left(D^{2}(f) ; \mathrm{Q}\right)$. By [7] Theorem 3.2 we have that

$$
\mu_{I}(f)=\frac{1}{2}\left\{\mu(X)+\mu\left(\left.X\right|_{y=0}\right)\right\} .
$$

But since $H$ and $\left.H\right|_{y=0}$ are both ICIS of dimension greater than 0 (this is because $p<2 n-1)$, we have that $\mu(H) \geq \tau(H)$ and $\mu\left(\left.H\right|_{y=0}\right) \geq \tau\left(\left.H\right|_{y=0}\right)$, with equality if $H$ is quasihomogeneous, (which is true if $f$ is quasihomogeneous). The theorem $\mu \geq \tau$ with equality if the spaces are quasihomogeneous is well known and is proved in [8].

By Lemma 2.6 we find

$$
\mu_{I} \geq \frac{1}{2}\left\{\operatorname{dim}_{\mathrm{C}} \frac{\mathcal{O}_{n}^{p-n}}{\left\langle y \frac{\partial H}{\partial y}, \frac{\partial H}{\partial x_{1}}, \ldots, \frac{\partial H}{\partial x_{n-1}}\right\rangle \mathcal{O}_{n}+I_{H} \mathcal{O}_{n}^{p-n}}\right\}
$$


with equality if $p=n+1$ and $H$ quasihomogeneous, (see Remark 2.10 (i)).

But

$$
\frac{\partial H}{\partial x}(x, y)=\frac{\partial\left(h\left(x, y^{2}\right)\right)}{\partial x}=\frac{\partial h}{\partial x}\left(x, y^{2}\right)
$$

and

$$
\frac{\partial H}{\partial y}(x, y)=\frac{\partial\left(h\left(x, y^{2}\right)\right)}{\partial y}=2 y \frac{\partial h}{\partial y}\left(x, y^{2}\right)
$$

Therefore,

$$
\begin{aligned}
\mu_{I} & \geq \frac{1}{2}\left\{\operatorname{dim}_{\mathrm{C}} \frac{\mathcal{O}_{n}^{p-n}}{\left\langle y^{2} \frac{\partial h}{\partial y}\left(x, y^{2}\right), \frac{\partial h}{\partial x_{1}}\left(x, y^{2}\right), \ldots, \frac{\partial h}{\partial x_{n-1}}\left(x, y^{2}\right)\right\rangle \mathcal{O}_{n}+I_{H} \mathcal{O}_{n}^{p-n}}\right\} \\
& =\frac{1}{2}\left\{2 \operatorname{dim}_{\mathrm{C}} \frac{\mathcal{O}_{n}^{p-n}}{\left\langle y \frac{\partial h}{\partial y}(x, y), \frac{\partial h}{\partial x_{1}}(x, y), \ldots, \frac{\partial h}{\partial x_{n-1}}(x, y)\right\rangle \mathcal{O}_{n}+I_{h} \mathcal{O}_{n}^{p-n}}\right\} \\
& =\operatorname{dim}_{\mathrm{C}} \frac{\mathcal{O}_{n}^{p-n}}{\left\langle y \frac{\partial h}{\partial y}(x, y), \frac{\partial h}{\partial x_{1}}(x, y), \ldots, \frac{\partial h}{\partial x_{n-1}}(x, y)\right\rangle \mathcal{O}_{n}+I_{h} \mathcal{O}_{n}^{p-n}} \\
& =\operatorname{cod}(f) \text { by Theorem } 2.8 .
\end{aligned}
$$

ReMARKs 2.12. (i) If the conjecture of Remark 2.10 (ii) holds then we can get an equality in the theorem when $f$ is quasihomogeneous. In fact this is the reason for conjecturing the statement to be true.

(ii) The assumption $p<2 n-1$ excludes the case that $H_{\mid y=0}$ is zero dimensional, because then $\mu \geq \tau$ is not necessarily true. However if $n=2$ and $p=3$ then $\left.H\right|_{y=0}$ is a hypersurface singularity (and in this case $\mu \geq \tau$ is true) and so the proof above gives $\mu_{I} \geq \operatorname{cod}(f)$ with equality if $f$ is quasihomogeneous. However, it is known that the Mond conjecture (see 2.7) holds in this case, see [10] and [16], and hence the result can be deduced from this.

The above theorem shows that one might expect a generalisation of the Mond conjecture to be true; i.e., the sum of the ranks of the Betti numbers of the reduced homology of the disentanglement is greater than or equal to the $\mathscr{A}_{e}$-codimension. However, this is not the case as the following example shows. Another counter example to this "generalisation" is given in [9], Example 3.26. 
EXAmPLE 2.13. If $p=2 n$, then the statement of the theorem is false; it is possible to have $\mu_{I}(f)<\operatorname{cod}(f)$.

Let $f:\left(\mathrm{C}^{2}, 0\right) \rightarrow\left(\mathrm{C}^{4}, 0\right)$ be given by

$$
f(x, y)=\left(x, y^{2}, y^{3}, x^{k} y\right),
$$

for any integer $k \geq 1$. This is the series $I I I_{k}$ in Kirk's thesis, [11]. Using Theorem 2.8 it is easy to calculate that $\operatorname{cod}(f)=2 k-1$ for all $k$.

Since the double point space, $D^{2}(f)$, is a quasihomogeneous ICIS we can calculate the Milnor number using weights and degrees, see also [7] Examples $4.16(1)$. We find that $\mu\left(D^{2}(f)\right)=2 k$ and as $\left.D^{2}(f)\right|_{y=0}=\emptyset$ we have

$$
\mu_{I}(f)=\frac{1}{2}\left\{\mu\left(D^{2}(f)\right)+\mu\left(\left.D^{2}(f)\right|_{y=0}\right)\right\}=k .
$$

Thus, $\mu_{I}(f)<\operatorname{cod}(f)$ for $k>1$.

\section{Augmentations.}

We now turn our attention to augmentations of map germs. Note that now we allow multigerms. For the one parameter unfolding $F$ of $f$ we will assume that $F$ can be written in the form $F(x, t)=(\tilde{F}(x, t), t)$, for some $\tilde{F}$.

\subsection{Preliminaries.}

Definition 3.1. Suppose $f:\left(\mathrm{C}^{n}, S\right) \rightarrow\left(\mathrm{C}^{p}, 0\right)$ can be unfolded by the map $F:\left(\mathrm{C}^{n} \times \mathrm{C}, S\right) \rightarrow\left(\mathrm{C}^{p} \times \mathrm{C}, 0\right)$, where $S$ is a finite set of points of $\mathrm{C}^{n}$. Let $g:\left(\mathrm{C}^{q}, 0\right) \rightarrow(\mathrm{C}, 0)$ be the germ of a holomorphic function. Then the augmentation of $f$ by $F$ and $g$, denoted $A_{F, g}(f)$, is the map germ,

$$
A_{F, g}(f):\left(\mathrm{C}^{n} \times \mathrm{C}^{q}, S \times 0\right) \rightarrow\left(\mathrm{C}^{p} \times \mathrm{C}^{q}, 0 \times 0\right),
$$

defined by

$$
A_{F, g}(f)(x, z)=(\tilde{F}(x, g(z)), z) .
$$

We also say that $f$ has been augmented by $F$ and $g$.

This notion was introduced by Goryunov in [6] and used by Cooper in [3] when $f$ is of $\mathscr{A}_{e}$-codimension one, $F$ is the $\mathscr{A}_{e}$-versal unfolding of $f$ and $g(z)=z^{2}$. Augmentations formed an essential part of his classification of $\mathscr{A}_{e}$-codimension one map germs. What we shall see is that more general augmentations are useful in classification, particularly for creating series of singularities.

EXAMPLES 3.2. (i) One of the simplest series of singularities is defined by 


$$
f(x)=\left(x^{2}, x^{2 k+1}\right)
$$

for some $k \geq 1$, see [2] p 58. Note that $\operatorname{cod}(f)=k$.

Then $F:\left(\mathrm{C}^{2}, 0\right) \rightarrow\left(\mathrm{C}^{3}, 0\right)$ given by $F(x, u)=\left(x^{2}, x^{2 k+1}+u x, u\right)$ is a stable unfolding of $f$. This is because $F$ has the same 2-jet as the 2-determined (stable) cross-cap.

Augmenting each singularity of the above series with this unfolding and the function $g(z)=z^{2}$ gives the $B_{k}$ series,

$$
(x, z) \mapsto\left(x^{2}, x^{2 k+1}+z^{2} x, z\right) .
$$

See [15]. Here $\operatorname{cod}(f)=k$.

By setting $k=1$ and augmenting $f$ with the unfolding $F$ and the series of functions $g(z)=z^{\mu+1}, \mu>0$ we get the $S_{\mu}$ series (this is Mond's notation, it is the $A_{\mu}$ series in [2]),

$$
(x, z) \mapsto\left(x^{2}, x^{3}+z^{\mu+1} x, z\right) .
$$

In this case we have $\operatorname{cod}(f)=\mu$.

(ii) Let $f:\left(\mathrm{C}^{n}, 0\right) \rightarrow(\mathrm{C}, 0)$ define an isolated hypersurface singularity. Then $F(x, u)=(f(x)+u l(x), u)$, where $l$ is a generic linear form on $\mathrm{C}^{n}$, is an unfolding of $F$ and so

$$
(x, z) \mapsto(f(x)+g(z) l(x), z)
$$

is an augmentation of $f$.

Note that both examples in (i) are folding maps and that $\operatorname{cod}\left(A_{F, g}(f)\right)$ is equal to $\operatorname{cod}(f) \tau(g)$. We shall be able to deduce the last fact from Theorem 3.3 .

Another important series in the Mond classification of maps from surfaces to three space is the $H_{k}$ series. Unfolding $f(x)=\left(x^{3}, x^{3 k-1}\right)$ with the mapping $F(x, u)=\left(x^{3}, x^{3 k-1}+u x, u\right)$ gives the $H_{k}$ series. This can be see as an augmentation if we assume that $g$ is the submersion $g(z)=z$. Unfortunately, with the results in this paper we shall not be able to say anything about this interesting series of examples.

From these examples it seems that augmentations could be of use in the study of series. The existence of series is controversial; no-one has yet produced a reasonable definition for a series. Nevertheless, they appear in concrete examples such as above. As we shall see, we can take any map germ of $\mathscr{A}_{e}$-codimension one and can augment with the $A_{k}$ or $D_{k}$ series of hypersurface singularities for example and produce new series of finitely determined singularities. 


\section{2. $\mathscr{A}_{e}$-Codimension of Augmentations.}

In this part we prove some results on the $\mathscr{A}_{e}$-codimension of an augmentation in the case that $F$ is a stable unfolding of $f$, i.e. $F$ is stable as a map germ. Note that this is not equivalent to $\operatorname{cod}(f)=1$, see Examples 3.1.

We begin with the main theorem of this section.

Theorem 3.3. Suppose the unfolding $F$ of $f$ is stable as a map germ. Then

$$
\operatorname{cod}(f) \tau(g) \leq \operatorname{cod}\left(A_{F, g}(f)\right) .
$$

If $F$ or $g$ is a quasihomogeneous map germ, then $\operatorname{cod}(f) \tau(g)=\operatorname{cod}\left(A_{F, g}(f)\right)$.

Proof. We use Damon's equality of $\mathscr{A}_{e}$-codimension and $\mathscr{K}_{V, e}$-codimension, see Theorem 1 of [4]. For ease of proof we shall change notation slightly. For $f:\left(\mathrm{C}^{n}, S\right) \rightarrow\left(\mathrm{C}^{p}, 0\right)$ we unfold with $F:\left(\mathrm{C}^{n+1}, S\right) \rightarrow\left(\mathrm{C}^{p+1}, 0\right)$ but now write $A_{F, g}(f)$ with $\mathrm{C}^{q}$ on the left of $\mathrm{C}^{n+1}$.

Let $F^{\prime}:\left(\mathrm{C}^{q} \times \mathrm{C}^{n+1}, 0 \times S\right) \rightarrow\left(\mathrm{C}^{q} \times \mathrm{C}^{p+1}, 0 \times 0\right)$ be the obvious trivial extension of $F$. This map is stable since $F^{\prime}$ is. Furthermore we can describe Derlog $\left(V^{\prime}\right)$ in terms of Derlog $(V)$, where $V$ and $V^{\prime}$ are the discriminants of $F$ and $F^{\prime}$ respectively. (Thus, for $n<p, V$ and $V^{\prime}$ are the images of the maps) That is, Derlog $\left(V^{\prime}\right)$ is generated over $\mathcal{O}_{V^{\prime}}$ by $\operatorname{Derlog}(V)$ and $\left\{e_{i}\right\}$, where $e_{i}$ is the $i$ th basis vector for $0_{q+p+1}^{q+p+1}, i=1, \ldots, q$, and $\operatorname{Derlog}(V)$ is identified with its image via the inclusion

$$
\text { inc: } \mathcal{O}_{p+1}^{p+1} \rightarrow \mathcal{O}_{q+p+1}^{q+p+1}, \quad \xi \mapsto(0, \xi) .
$$

The $\operatorname{map} f$ is the pullback via the map, $\Psi_{f}: \mathrm{C}^{p} \rightarrow \mathrm{C}^{q+p+1}$,

$$
\Psi_{f}\left(Y_{1}, \ldots, Y_{p}\right)=\left(0, \ldots, 0, Y_{1}, \ldots, Y_{p}, 0\right) .
$$

The map $F$ is the pullback via the map, $\Psi_{F}: \mathrm{C}^{p+1} \rightarrow \mathrm{C}^{q+p+1}$,

$$
\Psi_{F}\left(Y_{1}, \ldots, Y_{p}, U\right)=\left(0, \ldots, 0, Y_{1}, \ldots, Y_{p}, U\right) .
$$

The map $A_{F, g}(f)$ is the pullback via the map, $\Psi_{A}: \mathrm{C}^{p+q} \rightarrow \mathrm{C}^{q+p+1}$,

$$
\Psi_{A}\left(Z_{1}, \ldots, Z_{q}, Y_{1}, \ldots, Y_{p}\right)=\left(Z_{1}, \ldots, Z_{q}, Y_{1}, \ldots, Y_{p}, g(Z)\right) .
$$

Denote elements of Derlog $(V)$ by $\eta$ and those of Derlog $\left(V^{\prime}\right)$ by $\eta^{\prime}$. Choose a finite set $\left\{\eta_{r}^{\prime}\right\}$ of generators of Derlog $\left(V^{\prime}\right)$. Define $a_{r}$ to be the last row of the vector $\eta_{r}^{\prime}$ for all $r$.

It is trivial that $\frac{\partial \Psi_{f}}{\partial Y_{j}}=e_{q-j}$ for $j=1, \ldots, p$ and that $e_{i} \in \Psi_{f}^{*}\left(\operatorname{Derlog}\left(V^{\prime}\right)\right)$ for $i=1, \ldots, q$. Thus $T K_{V^{\prime}, e}\left(\Psi_{f}\right)$ contains $e_{i}$ for $i=1, \ldots, q+p$ and so 